\title{
Comparison of the regeneration of cartilage and the clinical outcomes after the open wedge high tibial osteotomy with or without microfracture: a retrospective case control study
}

\author{
O-Sung Lee', Seung Hoon Lee ${ }^{2}$, Su Jung Mok ${ }^{3}$ and Yong Seuk Lee ${ }^{3^{*}}$ (D)
}

\begin{abstract}
Background: It is unclear whether postoperative outcomes are associated with the cartilage regeneration after open wedge high tibial osteotomy (OWHTO) combined with microfracture. The purpose of this study was to evaluate the regeneration of the articular cartilage, radiologic, and clinical outcomes after OWHTO with and without microfracture.

Methods: Eighty-seven patients who underwent OWHTO from 2014 to 2015 were retrospectively included in this study. Fifty-seven OWHTOs with microfracture on medial femoral condyle (MFC) (group 1) and 30 OWHTOs without microfracture (group 2) were compared at a mean 2-year follow-up. The regeneration of the articular cartilage was evaluated using International Cartilage Repair Society (ICRS) grade on the second-look arthroscopy and the magnetic resonance observation of cartilage repair tissue (MOCART) score on magnetic resonance imaging (MRI). The weight-bearing line (WBL) ratio, hip-knee-ankle (HKA) angle, joint line convergence angle (JLCA) and Ahlbäck grade were evaluated. The clinical outcomes were evaluated using the Western Ontario and McMaster University (WOMAC) scores and the Knee Society (KS).
\end{abstract}

Results: The articular cartilage in the MFC were regenerated in 67.8\% of group 1 (43/57) and 58.6\% of group 2 (16/ $30)$, respectively $(p=0.014)$. However, change of the ICRS grades of the medial tibial plateau, lateral and patellofemoral compartments showed no statistical difference between the groups. Total MOCART score in group 1 was superior to that in the group 2 at postoperative 2 years ( $41.8 \pm 18.6$ vs. $31.8 \pm 19.8, p=0.023$ ). Regarding MOCART score, microfracture was only effective in the defect filling and integration to the border zone of the MFC $(p<0.001$ and $p=0.035$, respectively). Other radiologic and clinical outcomes showed no statistical differences between the groups.

Conclusion: Microfracture of the MFC during OWHTO only helped the filling of the degenerative cartilage defect and the integration of the cartilage with adjacent cartilage. However, the clinical and radiologic outcome could not be improved by mircrofracture in the OWHTO.

Keywords: Knee, Open wedge high tibial osteotomy, Microfracture, Cartilage, Regeneration

\footnotetext{
* Correspondence: smcos1@daum.net; smcos1@snu.ac.kr

${ }^{3}$ Department of Orthopaedic Surgery, Seoul National University College of

Medicine, Bundang Hospital, Seoul, South Korea

Full list of author information is available at the end of the article
}

(c) The Author(s). 2019 Open Access This article is distributed under the terms of the Creative Commons Attribution 4.0 International License (http://creativecommons.org/licenses/by/4.0/), which permits unrestricted use, distribution, and reproduction in any medium, provided you give appropriate credit to the original author(s) and the source, provide a link to the Creative Commons license, and indicate if changes were made. The Creative Commons Public Domain Dedication waiver (http://creativecommons.org/publicdomain/zero/1.0/) applies to the data made available in this article, unless otherwise stated. 


\section{Background}

Open wedge (OW) high tibial osteotomy (HTO) is an effective surgical treatment for patients with medial compartmental osteoarthritis combined with varus alignment [1-4]. The correction of the varus alignment by OW HTO provides change of the load distribution in the knee joint and improved functionality in patients with medial compartment osteoarthritic knees [5, 6]. Additionally, reduced load on the medial compartment due to lateral shift of the axial load has been shown to lead to biological regeneration of the articular cartilage after OW HTO [7-9]. However, lateral and patellofemoral compartments could be affected and changes of the cartilage status of these compartments are also questionable.

Although excellent short- to mid-term results after OW HTO have been reported in terms of clinical and radiologic results, clinical and radiological deterioration has been observed over long-term follow-up [2, 10, 11]. Therefore, several studies have suggested that OW HTO combined with cartilage repair techniques such as microfracture, subchondral drilling, abrasion arthroplasty, and autologous chondrocyte implantation might enable more effective cartilage regeneration and improved long-term outcomes [12-15]. Among them, microfracture has been one of the most commonly used procedures for cartilage repair procedures [14, 16-18]. Mocrofracture creates multiple holes in the subchondral bone in order to stimulate bone marrow. The cartilage defects are filled with precursor cells, resulting in a new cartilage and regenerative tissue [14]. However, it is unclear whether postoperative clinical outcomes are associated with the quality of cartilage regeneration after OW HTO combined with microfracture. Furthermore, little is known about the factors that influence clinical outcomes after OW HTO.

The purpose of this study was to compare the regeneration of the articular cartilage, radiologic, and clinical outcomes after OW HTO with and without microfracture. Our hypotheses were that the articular cartilage in the medial compartment after OW HTO with microfracture would be regenerated better than that without microfracture regardless of the radiologic and clinical outcomes of both groups [19].

\section{Methods}

\section{Patients}

This retrospective case-case control study was carried out between between March 2014 and May 2015. Patients received an arthroscopy at the time of OW HTO. We performed microfracture only when the medial femoral condyle (MFC) hadd a full-thickness articular cartilage lesion with a ballotable and unstable flap of cartilage or a partial thickness articular cartilage lesion of which the cartilage was simply scraped off down to the bone when probed. Microfracture was not performed if the patient had an overly large full-thickness lesion with an evenly distributed and stable calcified layer or if the surrounding cartilage was stable. All patients underwent removal of the locking plate with second-look arthroscopy at around 2 years after OW HTO. This study included only patients who completed radiologic and clinical assessment at the time of locking plate removal.

The inclusion criteria for OW HTO were: (1) primary osteoarthritis (not inflammatory arthritis), (2) radiographs showing medial-compartment knee osteoarthritis of Ahlbäck grades 1 to 3, [20] (3) concurrent varus alignment of operated limb, and (4) the patients who failed to respond to non-surgical treatments such as weight loss, physical therapy, activity modification, and drugs.

From March 2014 to May 2015, a total of 124 knees underwent OW HTO. Among them, 17 patients excluded from this study due to our exclusion criteria and inadequate medical records: (1) secondary arthritis such as post-traumatic arthritis, (2) OW HTO associated with additional surgery such as anterior cruciate ligament reconstruction, (3) concurrent bilateral OW HTO, (4) revision OW HTO, (5) double osteotomy including distal femoral osteotomy, and (6) the patient whose standing radiograph in full knee extension could not be obtained because of the limitation of extension. Additionally, we couldn't conduct second-look arthroscopy in 14 patients and 6 patients were lost during follow-up period. Final drop-out rate was $29.8 \%$ and 87 patients were successfully included in this study. Fifty-seven OW HTOs with microfracture on the MFC (group1) and 30 OW HTOs without microfracture (group2) were compared with a mean follow-up of 2 years (mean $2.0 \pm 1.6$ ) (Fig. 1). This retrospective study obtained the approval of the institutional review board of our hospital.

\section{Surgical technique and rehabilitation}

All surgical procedures were performed by a single surgeon. The target mechanical axis was the weight-bearing line passing through $62.5 \%$ of the width of the tibial plateau, corresponding to a postoperative mechanical valgus of $2^{\circ}$ to $4^{\circ}$. All patients received an arthroscopy at the time of OW HTO. Arthroscopic procedures included joint debridement, meniscectomy of a medial meniscus tear, removal of loose bodies and microfracture. We trimmed any loose flaps of articular cartilage and debrided the subchondral bone. The holes were made with an arthroscopic awl perpendicularly through the exposed subchondral bone, at which point bleeding from the holes was observed. After the microfracture, a longitudinal incision about 5-cm long was made at the anteromedial aspect of the proximal tibia. After release of the superior border of the pes anserinus and the anterior border of the medial collateral ligament, horizontal osteotomy was performed, and an additional biplanar anterior osteotomy was performed. The gradual distraction was performed at the most posterior portion of the osteotomy gap until the target limb alignment was obtained [21]. The osteotomized bone was fixed with a long 


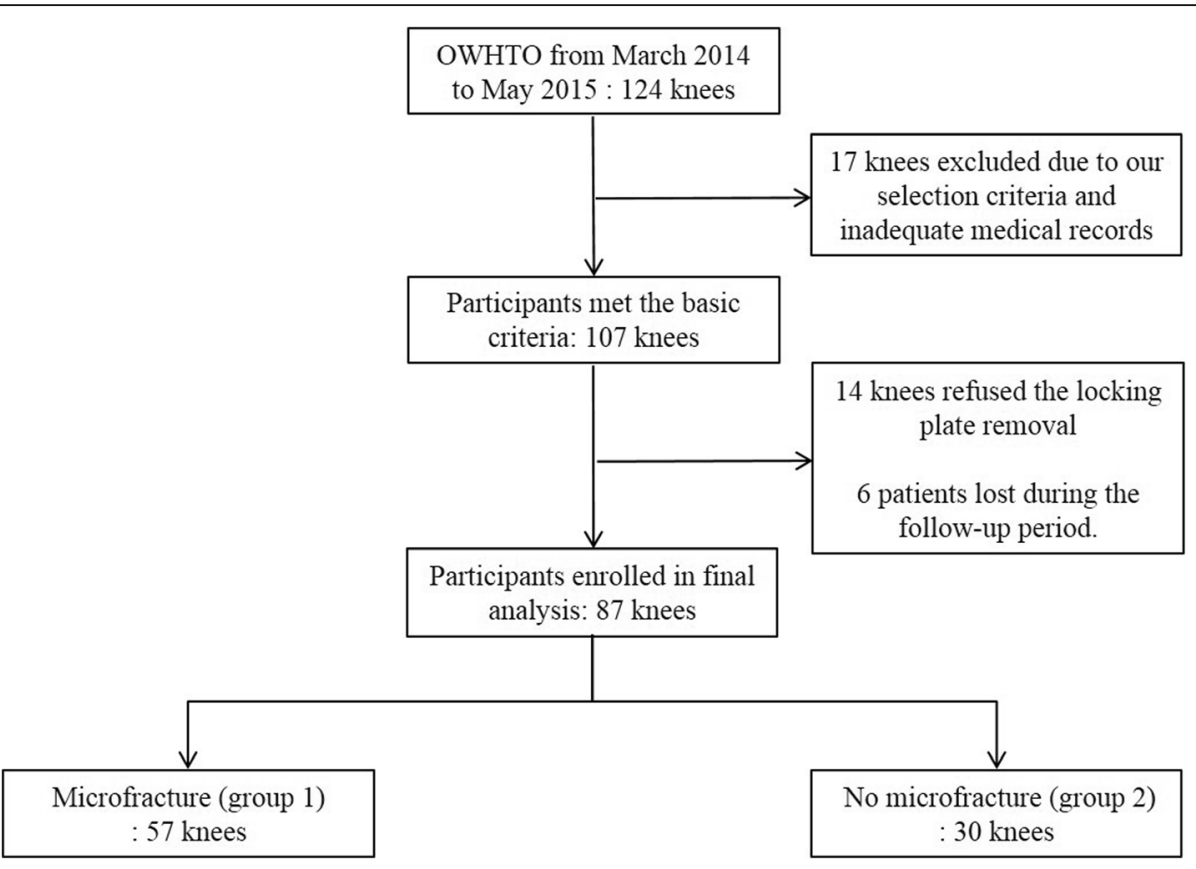

Fig. 1 Study cohort and design (OW HTO, open wedge high tibial osteotomy)

locking plate (DWLP, TDM, Seongnam, Korea). The osteotomy gap was then filled with a bone graft substitute and no intra-articular drain was inserted.

In terms of postoperative rehabilitation, tolerable weightbearing with crutches was encouraged for the patients with no microfracture, and they were allowed full weight-bearing, immediately postoperatively. However, the patients with microfracture treatment were allowed partial weight-bearing at postoperative 2 weeks and full weight-bearing at postoperative 4 weeks. All patients performed a passive exercise for range of motion of the operated knee at 2 days after surgery until a maximum flexion angle of $130^{\circ}$ or more was achieved. Squatting with weight was restricted for 3 months, and patients were instructed to be careful when getting up from the sitting position.

\section{Evaluation of cartilage regeneration}

The articular cartilage of the MFC, medial tibial plateau (MTP), lateral femoral condyle (LFC), lateral tibial plateau (LTP), patella, and trochlea was evaluated according to the International Cartilage Repair Society (ICRS) grading system by an arthroscopy at the time of OW HTO. The regeneration of the articular cartilage was also evaluated according to the ICRS grading system by second-look arthroscopy at the time of plate removal [22]. The ICRS grading system classified a macroscopically normal cartilage without notable defects as ICRS 0, a cartilage with a fibrillated, slightly softening surface or an superficial fissures as ICRS 1 , a defect < $50 \%$ of the cartilage thickness as ICRS 2 , a defect $>50 \%$ of the cartilage thickness as ICRS 3, and a full-thickness osteochondral injury as ICRS 4.

In addition, all patients underwent postoperative magnetic resonance imaging (MRI) to assess the quality of cartilage regeneration on the day before plate removal. Routine MRI protocol included fast-spin echo (dual T2-FSE) and fat-suppressed gradient echo (3D-GE-FS) sequences with 3-Tmagnetic resonance system (Ingenia, Philips Healthcare, Best, Netherlands). Morphological evaluation of the postoperative articular cartilage status on the MFC was performed according to a modified magnetic resonance observation of cartilage repair tissue (MOCART) scoring system by 2 independent investigators in a blinded manner [23]. A MOCART score of 100 indicates the best articular cartilage status and 0 indicates the worst status [24].

\section{Evaluation of radiologic and clinical outcomes}

Clinical and radiographic evaluations were performed preoperatively and at the time of second-look arthroscopy. The weight-bearing line (WBL) ratio, hip-knee-ankle (HKA) angle, joint line convergence angle (JLCA) and Ahlbäck grade were measured preoperatively and at the time of second-look arthroscopy on full-length standing anteroposterior radiographs. The INFINITT version 5.0.9.2 (INFINITT, Seoul, South Korea) was used for the radiographic measurements. The HKA angle was measured as the angle between the line from the center of the femoral head to the center of the knee joint and the line from the center of the knee joint to the center of the ankle joint. A WBL was defined as the line drawn from the center of the femoral head 
to the center of the superior surface of the talus. The denominator of WBL ratio was the width of the tibial plateau, and the numerator of WBL ratio was the medial tibial intersection of the WBL in the knee joint (the medial tibial edge at $0 \%$ and the lateral tibial edge at 100\%). The JLCA was defined as the angle between the line connecting the articular surfaces of the distal femur and the proximal tibia. Additionally, the Ahlbäck classification was used for the evaluation of the radiologic severity of osteoarthritis [20]. Flexion contracture and active maximal flexion were measured in the supine position using a goniometer. The clinical status of each knee was rated using the Western Ontario and McMaster University (WOMAC) scores and the Knee Society (KS) knee and functional scores.

\section{Statistical analysis}

Statistical analyses were performed with SPSS version 22.0 statistical package (IBM Corp., Armonk, NY, USA). Data was described based on means and standard deviation (SD) for continuous values. The differences in continuous variables were analyzed with Student's t-test or the Mann-Whitney test according to the appropriate normality tests. The differences of other categorical variables were analyzed with Pearson's chi-square test or Fisher exact test or linear by linear association. Univariate and multivariate regression analysis were performed to identify factors affecting the clinical results represented by KS knee and functional scores and WOMAC scores. All statistical significances were set at $p<0.05$.

We determined a total sample size using a post hoc power analysis based on a previous study similar to this study [25]. A 10-point difference on the Knee Society scores was considered significant. An error and power were set to 5 and $80 \%$, respectively using computer software $\left(G^{*}\right.$ Power 3.1.0). The required sample size required was 33 knees per group, considering a possible dropout rate of $30 \%$.

\section{Results}

Among a total of 87 patients included in this study, 57 patients were included in group 1 (with microfracture) and 30 OWHTOs were included in group 2 (without microfracture), with a mean follow-up of two years. Both groups showed no statistical differences in terms of preoperative demographics. Additionally, no statistical differences between groups were observed with respect to WBL ratio, HKA angle, JLCA, Ahlbäck grade of osteoarthritis, range of motion, WOMAC scores and Knee scores (Table 1).

The preoperative and postoperative assessments of articular cartilage according to the arthroscopic examinations are summarized in Table 2. The improvement of ICRS grade of MFC was observed in 75.4\% (43/57) among the patients of group 1, whereas $53.3 \%(16 / 30)$ among the patients of group 2 showed the improvement of ICRS grade of MFC ( $p=0.014)$. The improvement of MTP was observed in $61.4 \%(35 / 57)$ of group 1 and $53.3 \%(15 / 30)$ of group 2 with no statistical difference $(p=0.742)$. Otherwise, the articular cartilages of the LFC deteriorated in $38.6 \%$ (22/57) of group 1 and $26.9 \%$ (8/30) of group 2, respectively $(p=0.674)$, and that of the LTP deteriorated in $36.8 \%$ (21/57) of group 1 and $40.0 \%$ (12/30) of group 2, respectively $(p=0.936)$. Additionally, the articular cartilages of the patella and trochlea also showed similar deterioration (group 1 vs. group 2; $47.4 \%$ vs. $40.0 \%, p=0.480$; $45.6 \%$ vs. $63.3 \%, p=0.496$, respectively).

The mean MOCART score of the MFC mean at postoperative 2 years was superior in group 1 compared to that in the group $2(41.8 \pm 18.6$ vs. $31.8 \pm 19.8, p=0.023)$. The number of knees showing complete filling, hypertrophy, $>50 \%$ of adjacent cartilage, $<50 \%$ of adjacent cartilage, subchondral bone exposure were 14, 16, 9, 15, 3 in group 1 and 2, 1, 5, 6, 16 in group 2, respectively $(p<0.001)$. In terms of the integration of repaired cartilage to the border zone, the number of knees showing complete integration, visible demarcating border, defect $<50 \%$ of the length of repair tissue, defect $>50 \%$ of the length of repair tissue were 3,29, 16, 9 in group 1 and 3, $6,9,12$ in group 2 , respectively $(p=0.035)$ (Table 3$)$. Although the microfracture of the MFC appeared to be helpful for cartilage healing according to the MOCART score, it was helpful only for the degree of defect repair and the integration to the border zone excluding other variables.

There were no statistically significant differences of the WBL ratio, HKA and JLCA between groups. Moreover, the severity of osteoarthritis indicated by the Ahlbäck grading system showed no statistical difference between groups $(p=0.699)$. All clinical scores showed no statistical difference between groups at a mean 2-year follow-up (Table 4).

The results of univariate and multivariate regression analysis for evaluating factors affecting clinical scores after OW HTO are shown in Table 5. The univariate analysis showed that the postoperative values of HKA angle, WBL ratio, JLCA, Ahlbäck grade and postoperative cartilage healing of MFC and MTP were significantly related with the postoperative clinical scores at a mean 2-year follow-up. However, in mulivariate analysis, only postoperative WBL ratio was significantly associated with postoperative KS knee score and WOMAC pain score ( $p=0.010$ and $p=0.045$, respectively).

\section{Discussion}

The principal findings of this study were that microfracture of the MFC during OW HTO improved the regeneration of the articular cartilage of the MFC, whereas those in the lateral and patellofemoral compartments 
Table 1 Comparison of preoperative demographics and measurements

\begin{tabular}{|c|c|c|c|c|}
\hline \multicolumn{2}{|l|}{$\bar{l}$} & Microfracture $(n=57)$ & No microfracture $(n=30)$ & $p$-value \\
\hline \multicolumn{2}{|l|}{ Patients number } & 57 & 30 & \\
\hline \multicolumn{2}{|l|}{ Age (years) } & $57.0 \pm 5.4$ & $57.0 \pm 6.5$ & $0.989^{*}$ \\
\hline \multicolumn{2}{|c|}{ Sex (male/female) } & $37 / 20$ & $7 / 23$ & $0.333 \S$ \\
\hline \multicolumn{2}{|l|}{ Side (Left: Right) } & $22 / 35$ & $15 / 15$ & $0.365 \S$ \\
\hline \multicolumn{2}{|l|}{ Height (cm) } & $159.1 \pm 8.1$ & $156.8 \pm 8.9$ & $0.229^{*}$ \\
\hline \multicolumn{2}{|l|}{ Weight (kg) } & $67.4 \pm 12.6$ & $62.2 \pm 10.2$ & $0.403^{*}$ \\
\hline \multicolumn{2}{|c|}{ Body mass index $\left(\mathrm{kg} / \mathrm{m}^{2}\right)$} & $26.5 \pm 3.6$ & $26.4 \pm 3.3$ & $0.950^{*}$ \\
\hline \multicolumn{2}{|c|}{ Onset of symptom (months) } & $34.7 \pm 14.3$ & $36.1 \pm 25.3$ & $0.897^{*}$ \\
\hline \multicolumn{2}{|l|}{ Follow up (years) } & $2.0 \pm 0.2$ & $1.9 \pm 0.1$ & $0.300^{*}$ \\
\hline \multicolumn{2}{|c|}{ Weight bearing line ratio (\%) } & $19.1 \pm 11.7$ & $22.0 \pm 12.3$ & $0.153^{*}$ \\
\hline \multicolumn{2}{|c|}{ Hip-knee-ankle angle $\left(^{\circ}\right)$} & Varus $7.0 \pm 3.1$ & Varus $6.2 \pm 2.7$ & $0.241^{*}$ \\
\hline \multicolumn{2}{|c|}{ Joint line convergence angle $\left({ }^{\circ}\right)$} & $3.1 \pm 1.7$ & $2.8 \pm 1.4$ & $0.545^{*}$ \\
\hline \multicolumn{2}{|c|}{ Ahlbäck grade (grade 0/1/2/3/4) } & $0 / 42 / 13 / 2 / 0$ & 0/26/4/0/0 & $0.162 \dagger$ \\
\hline \multicolumn{2}{|c|}{ Flexion contracture $\left(^{\circ}\right)$} & $2.5 \pm 1.4$ & $1.8 \pm 2.8$ & $0.393^{*}$ \\
\hline \multicolumn{2}{|c|}{ Active full flexion $\left({ }^{\circ}\right)$} & $132.1 \pm 7.2$ & $134.0 \pm 4.8$ & $0.201^{*}$ \\
\hline \multirow[t]{4}{*}{ WOMAC scores } & Total & $39.8 \pm 13.2$ & $39.5 \pm 7.8$ & $0.914^{*}$ \\
\hline & Pain & $9.5 \pm 3.5$ & $8.4 \pm 2.6$ & $0.278^{*}$ \\
\hline & Stiffness & $4.3 \pm 2.1$ & $4.2 \pm 2.0$ & $0.804^{*}$ \\
\hline & Function & $26.0 \pm 9.7$ & $26.9 \pm 8.7$ & $0.674^{*}$ \\
\hline \multirow[t]{2}{*}{ Knee scores } & Knee scores & $53.7 \pm 17.0$ & $52.7 \pm 14.3$ & $0.791^{*}$ \\
\hline & Function scores & $59.5 \pm 15.5$ & $59.8 \pm 9.0$ & $0.925^{*}$ \\
\hline
\end{tabular}

The values are presented as mean \pm standard deviation. *Derived using the Student's $\mathrm{t}$-test. $\S$ Derived using the Pearson chi-square test. + Derived using the linear by linear association WOMAC; Western Ontario and McMaster Universities

Table 2 Change in International Cartilage Research Society grade

\begin{tabular}{|c|c|c|c|c|c|}
\hline \multirow{2}{*}{$\begin{array}{l}\text { ICRS grade on second look } \\
\text { arthroscopy }\end{array}$} & \multicolumn{2}{|c|}{ Microfracture $(n=57)$} & \multicolumn{2}{|c|}{ No microfracture $(n=30)$} & \multirow[t]{2}{*}{$p$-value } \\
\hline & Preoperative & Postoperative & Preoperative & Postoperative & \\
\hline MFC (Grade 0/1/2/3/4) & $0 / 3 / 13 / 21 / 20$ & $3 / 8 / 9 / 15 / 8 / 14$ & $0 / 2 / 7 / 15 / 6$ & $0 / 7 / 15 / 6 / 2 / 0$ & \\
\hline Improvement/stationary/worsening & \multicolumn{2}{|c|}{$43(75.4 \%) / 13(22.8 \%) / 1(1.8 \%)$} & \multicolumn{2}{|c|}{$16(53.3 \%) / 10(33.3 \%) / 4(13.3 \%)$} & $0.014^{*}$ \\
\hline MTP (Grade 0/1/2/3/4) & $0 / 0 / 18 / 6 / 33$ & $1 / 11 / 22 / 13 / 9 / 1$ & $0 / 5 / 12 / 10 / 3$ & $1 / 11 / 10 / 5 / 3 / 0$ & \\
\hline Improvement/stationary/worsening & \multicolumn{2}{|c|}{35 (61.4\%) / 15 (26.3\%) / 7 (12.3\%) } & \multicolumn{2}{|c|}{$15(50.0 \%) / 13(43.3 \%) / 2(6.7 \%)$} & $0.742^{*}$ \\
\hline LFC (Grade 0/1/2/3/4) & $21 / 32 / 4 / 0 / 0$ & $7 / 38 / 12 / 0 / 0$ & $17 / 10 / 3 / 0 / 0$ & $9 / 18 / 3 / 0 / 0$ & \\
\hline Improvement/stationary/worsening & \multicolumn{2}{|c|}{$3(5.3 \%) / 32(56.1 \%) / 22$ (38.6\%) } & \multicolumn{2}{|c|}{0 (0.0\%) / 22 (73.3\%) / 8 (26.7\%) } & $0.674^{*}$ \\
\hline LTP (Grade 0/1/2/3/4) & $8 / 42 / 7 / 0 / 0$ & $1 / 36 / 18 / 2 / 0$ & $8 / 21 / 1 / 0 / 0$ & 6/13/11/0/0 & \\
\hline Improvement/stationary/worsening & \multicolumn{2}{|c|}{$3(5.3 \%) / 33(57.9 \%) / 21$ (36.8\%) } & \multicolumn{2}{|c|}{2 (6.7\%) / $16(53.3 \%) / 12$ (40.0\%) } & $0.936^{*}$ \\
\hline Patella (Grade 0/1/2/3/4) & $15 / 29 / 9 / 2 / 2$ & $1 / 29 / 22 / 4 / 1$ & $9 / 16 / 4 / 1 / 0$ & $4 / 14 / 11 / 1 / 0$ & \\
\hline Improvement/stationary/worsening & \multicolumn{2}{|c|}{$4(7.0 \%) / 26(45.6 \%) / 27(47.4 \%)$} & \multicolumn{2}{|c|}{$3(10.0 \%) / 15(50.0 \%) / 12(40.0 \%)$} & $0.480^{*}$ \\
\hline Trochlea (Grade 0/1/2/3/4) & $7 / 26 / 18 / 5 / 1$ & $2 / 15 / 29 / 9 / 2$ & $9 / 12 / 6 / 3 / 0$ & $2 / 7 / 16 / 5 / 0$ & \\
\hline Improvement/stationary/worsening & \multicolumn{2}{|c|}{$4(7.0 \%) / 27(47.4 \%) / 26(45.6 \%)$} & \multicolumn{2}{|c|}{$4(13.3 \%) / 7(23.3 \%) / 19(63.3 \%)$} & $0.496^{*}$ \\
\hline
\end{tabular}

The values are presented as number with the percent in parentheses. * Derived using the linear by linear association. The statistical significance was set at $p<0.05$ derived using the linear by linear association. MFC medial femoral condyle, MTP medial tibial plateau, LFC lateral femoral condyle, LTP lateral tibial plateau 
Table 3 Comparison of MOCART scores of medial femoral condyle between the microfracture group and no-microfracture group using MRI

\begin{tabular}{|c|c|c|c|c|c|}
\hline \multicolumn{3}{|l|}{ Variable } & Microfracture $(n=57)$ & No microfracture $(n=30)$ & $p$-value \\
\hline \multicolumn{3}{|l|}{ Total score } & $41.8 \pm 18.6$ & $31.8 \pm 19.8$ & $0.023^{*}$ \\
\hline \multirow[t]{6}{*}{ 1. Degree of defect repair and filling of the defect } & \multicolumn{2}{|l|}{ Complete } & 14 & 2 & $<0.001 \dagger$ \\
\hline & \multicolumn{2}{|l|}{ Hypertrophy } & 16 & 1 & \\
\hline & \multicolumn{2}{|l|}{ Incomplete } & & & \\
\hline & \multicolumn{2}{|c|}{$>50 \%$ of adjacent cartilage } & 9 & 5 & \\
\hline & \multicolumn{2}{|c|}{$<50 \%$ of adjacent cartilage } & 15 & 6 & \\
\hline & \multicolumn{2}{|c|}{ Subchondral bone exposed } & 3 & 16 & \\
\hline \multirow[t]{5}{*}{ 2. Integration to the border zone } & \multicolumn{2}{|l|}{ Complete } & 3 & 3 & $0.035+$ \\
\hline & \multicolumn{2}{|l|}{ Incomplete } & & & \\
\hline & \multicolumn{2}{|c|}{ Demarcating border visible } & 29 & 6 & \\
\hline & \multicolumn{2}{|c|}{$\begin{array}{l}\text { Defect }<50 \% \text { of the length of repair } \\
\text { tissue }\end{array}$} & 16 & 9 & \\
\hline & \multicolumn{2}{|c|}{$\begin{array}{l}\text { Defect }>50 \% \text { of the length of repair } \\
\text { tissue }\end{array}$} & 9 & 12 & \\
\hline \multirow[t]{3}{*}{ 3. Surface of the repair tissue } & \multicolumn{2}{|c|}{ Surface intact } & 12 & 4 & $0.115+$ \\
\hline & \multicolumn{2}{|c|}{$\begin{array}{l}\text { Surface damaged }<50 \% \text { of repair } \\
\text { tissue depth }\end{array}$} & 29 & 12 & \\
\hline & \multicolumn{2}{|c|}{$\begin{array}{l}\text { Surface damaged }>50 \% \text { of repair } \\
\text { tissue depth }\end{array}$} & 16 & 14 & \\
\hline \multirow[t]{2}{*}{ 4. Structure of the repair tissue } & \multicolumn{2}{|c|}{ Homogeneous } & 7 & 4 & $0.998 \S$ \\
\hline & \multicolumn{2}{|c|}{ Inhomogeneous or cleft formation } & 50 & 26 & \\
\hline \multirow[t]{6}{*}{ 5. Signal intensity of the repair tissue } & \multirow[t]{3}{*}{ Dual T2-FSE } & Isointense & 3 & 0 & $0.311+$ \\
\hline & & Moderately hypointense & 17 & 8 & \\
\hline & & Markedly hypointense & 37 & 22 & \\
\hline & \multirow[t]{3}{*}{ 3D-GE-FS } & Isointense & 6 & 2 & $0.396+$ \\
\hline & & Moderately hypointense & 21 & 9 & \\
\hline & & Markedly hypointense & 30 & 19 & \\
\hline \multirow[t]{2}{*}{ 6. Subchondral lamina } & \multicolumn{2}{|l|}{ Intact } & 32 & 23 & $0.066 \S$ \\
\hline & \multicolumn{2}{|l|}{ Not intact } & 25 & 7 & \\
\hline \multirow[t]{2}{*}{ 7. Subchondral bone } & \multicolumn{2}{|l|}{ Intact } & 40 & 28 & $0.061 \S$ \\
\hline & Edema & & 17 & 2 & \\
\hline 8. Adhesions & No & & 49 & 28 & $0.483 \S$ \\
\hline & Yes & & 8 & 2 & \\
\hline 9. Effusion & No & & 48 & 22 & $0.261 \S$ \\
\hline & Yes & & 9 & 8 & \\
\hline
\end{tabular}

The values are presented as number except for the total score indicated. *Derived using the Student's t-test. †Derived using the linear by linear association. $\S$ Derived with Pearson chi-square test. The statistical significance was set at $p<0.05$. MOCART magnetic resonance observation of cartilage repair tissue

were found to have deteriorated at a mean 2-year follow-up. It was only effective with respect to the degree of defect repair (filling of the defect) and integration to the border zone of the MFC. Additionally, microfracture could not improve the radiologic and clinical outcomes after the OW HTO.

Several authors reported the regeneration of degenerated articular cartilage after HTO in the absence of any cartilage repair procedure $[8,9,26,27]$. Fujisawa et al.
[26] reported that the cartilage lesion was covered with fibrous and membranous tissue about 1.5 to 2 years after osteotomy if ideal correction was obtained. Koshino et al. [9] reported that 133 of 146 cases (91\%) achieved partial or total coverage with fibrocartilage after closed wedge HTO. However, cartilage repair procedures such as microfracture, subchondral drilling, and abrasion arthroplasty in conjunction with HTO have reportedly contributed to more favorable clinical and histological 
Table 4 Comparison of postoperative results between microfracture group and no microfracture group

\begin{tabular}{|c|c|c|c|c|}
\hline \multirow{2}{*}{\multicolumn{2}{|c|}{ Weight bearing line ratio (\%) }} & Microfracture $(n=57)$ & No microfracture $(n=30)$ & $p$ value \\
\hline & & $58.6 \pm 7.2$ & $57.5 \pm 6.2$ & $0.060^{*}$ \\
\hline \multicolumn{2}{|c|}{ Hip-knee-ankle angle $\left(^{\circ}\right)$} & Valgus $2.3 \pm 1.4$ & Valgus $1.9 \pm 1.6$ & $0.270^{*}$ \\
\hline \multicolumn{2}{|c|}{ Joint line convergence angle $\left({ }^{\circ}\right)$} & $1.9 \pm 1.7$ & $2.1 \pm 2.1$ & $0.705^{*}$ \\
\hline \multicolumn{2}{|c|}{ Ahlbäck grade (grade 0/1/2/3/4) } & $11 / 2 / 38 / 7 / 1$ & $5 / 23 / 2 / 0 / 0$ & $0.699+$ \\
\hline \multicolumn{2}{|c|}{ Flexion contracture $\left(^{\circ}\right)$} & $2.2 \pm 1.2$ & $1.8 \pm 1.6$ & $0.403^{*}$ \\
\hline \multicolumn{2}{|l|}{ Further flexion $\left(^{\circ}\right)$} & $134.9 \pm 7.2$ & $136.0 \pm 6.6$ & $0.492^{*}$ \\
\hline \multirow[t]{4}{*}{ WOMAC scores } & Total & $9.2 \pm 6.1$ & $10.2 \pm 7.8$ & $0.513^{*}$ \\
\hline & Pain & $1.3 \pm 1.2$ & $1.7 \pm 1.6$ & $0.278^{*}$ \\
\hline & Stiffness & $1.1 \pm 1.4$ & $1.3 \pm 1.5$ & $0.433^{*}$ \\
\hline & Function & $6.7 \pm 4.5$ & $7.2 \pm 5.9$ & $0.678^{*}$ \\
\hline \multirow[t]{2}{*}{ KS scores } & KS knee scores & $89.1 \pm 10.7$ & $88.3 \pm 10.8$ & $0.745^{*}$ \\
\hline & KS function scores & $88.3 \pm 10.8$ & $86.1 \pm 12.3$ & $0.634^{*}$ \\
\hline
\end{tabular}

The values are presented as mean \pm standard deviation. *Derived using the Student's t-test. †Derived using the linear by linear association. WOMAC Western Ontario and McMaster Universities

Table 5 Regression analysis of clinical scores

\begin{tabular}{|c|c|c|c|c|c|c|}
\hline Univariate regression analysis & KS knee & KS function & WOMAC pain & WOMAC stiffness & WOMAC function & WOMAC total \\
\hline Age & 0.809 & 0.539 & 0.549 & 0.962 & 0.585 & 0.639 \\
\hline BMl & 0.476 & 0.204 & 0.207 & 0.776 & 0.277 & 0.188 \\
\hline Sex & 0.398 & 0.597 & 0.075 & 0.259 & 0.877 & 0.972 \\
\hline Duration of symptom & 0.906 & 0.599 & 0.07 & 0.455 & 0.751 & 0.629 \\
\hline Site of microfracture & 0.636 & 0.514 & 0.118 & 0.349 & 0.788 & 0.765 \\
\hline Size of microfracture & 0.244 & 0.729 & 0.568 & 0.300 & 0.629 & 0.804 \\
\hline Preoperative HKA angle & 0.982 & 0.95 & 0.447 & 0.818 & 0.204 & 0.27 \\
\hline Postoperative HKA angle & 0.003 & 0.002 & $<0.001$ & 0.52 & 0.053 & 0.006 \\
\hline Preoperative WBL ratio & 0.873 & 0.903 & 0.183 & 0.828 & 0.093 & 0.106 \\
\hline Postoperative WBL ratio & $<0.001$ & $<0.001$ & $<0.001$ & 0.396 & 0.089 & 0.009 \\
\hline Preoperative JLCA & 0.069 & 0.057 & 0.677 & 0.703 & 0.926 & 0.919 \\
\hline Postoperative JLCA & 0.031 & 0.006 & 0.002 & 0.73 & 0.122 & 0.077 \\
\hline Preoperative Ahlbäck grade & 0.495 & 0.356 & 0.607 & 0.726 & 0.284 & 0.28 \\
\hline Postoperative Ahlbäck grade & 0.042 & 0.073 & 0.089 & 0.964 & 0.526 & 0.382 \\
\hline Cartilage healing of MFC & 0.001 & 0.001 & $<0.001$ & 0.443 & 0.296 & 0.098 \\
\hline Cartilage healing of MTP & 0.004 & 0.002 & 0.002 & 0.326 & 0.485 & 0.894 \\
\hline Multivariate regression analysis & KS knee & KS function & WOMAC pain & WOMAC total & & \\
\hline Postoperative HKA angel & 0.126 & 0.327 & 0.754 & 0.086 & & \\
\hline Postoperative WBL ratio & 0.010 & 0.06 & 0.045 & 0.892 & & \\
\hline Postoperative JLCA & 0.652 & 0.635 & 0.806 & & & \\
\hline Postoperative Ahlbäck grade & 0.654 & & & & & \\
\hline Cartilage healing of MFC & 0.055 & 0.184 & 0.089 & & & \\
\hline Cartilage healing of MTP & 0.582 & 0.337 & 0.686 & & & \\
\hline
\end{tabular}

HKA hip-knee-ankle, WBL weight bearing line, JLCA joint line convergence angle, MFC medial femoral condyle, MTP medial tibial plateau, KS Knee Society, WOMAC Western Ontario and McMaster Universities 
outcomes [14, 18, 25]. Among them, microfracture has been commonly performed with OW HTO by many surgeons because of its safety and convenience. However, the effect of microfracture on clinical outcomes has been controversial [14, 16-18]. In terms of clinical outcomes, Sterett et al. [28] reported excellent survival rates of 91\% survivorship at 7 years after microfracture with OW HTO. Pascale et al. [18] reported that patient satisfaction was increased among those who underwent HTO plus microfracture compared with those who underwent HTO alone at the 5-year follow-up. However, Matsunaga et al. [14] reported that microfracture combined with HTO did not lead to superior clinical outcomes compared to HTO alone at 1,3 , and 5 years postoperatively. Moreover, Ferruzzi et al. [16] reported that microfracture associated with HTO provided the worst Hospital for Special Surgery scores and WOMAC scores compared to both HTO alone and HTO with autologous chondrocyte implantation.

In terms of the macroscopic healing of the articular cartilage after OW HTO, second-look arthroscopies have been found to confirm the regeneration of degenerated articular cartilage in the medial compartment after HTO without any cartilage regeneration techniques $[8,9,29]$. Indeed, Jung et al. [8] reported that the regeneration of degenerated cartilage of the MFC was found in 75-92\% of patients after OW HTO without any cartilage regeneration strategies. Kim et al. [29] also reported that lesions in the MFC and the MTP of 104 HTO knees were improved in 54 knees (51.9\%) and 36 knees (34.6\%) without additional cartilage regeneration techniques. Moreover, Matsunaga et al. [14] reported that the second-look arthroscopy revealed better regeneration of the distal femur cartilage in the patients with the abrasion arthroplasty with HTO than those with either HTO alone $(p<0.0005)$ or microfracture with HTO $(p<0.01)$, with no difference between the 2 approaches.

Several articles have investigated the effect of simple HTO without any cartilage repair procedures on clinical outcomes [30, 31]. Spahn et al. [30] found that a patient history of more than 24 months, a poor preoperative clinical score, obesity, and smoking were associated with a worse outcome after HTO alone at midterm follow-up. In another study, age $>56$ years and postoperative knee flexion less than $120^{\circ}$ were significantly related to a poor outcome after simple HTO $(p=0.008$ and $p<0.001$, respectively), whereas the Ahlbäck grade of medial compartment arthritis and excellent preoperative KS scores were significantly related to a better outcome after simple HTO $(p<0.001$ and $p<0.001$, respectively) [31]. However, it has remained unclear which preoperative and postoperative factors affect clinical outcomes after HTO combined with a cartilage procedure. In our study, although no statistical results were obtained, a number of patients, whose cartilage
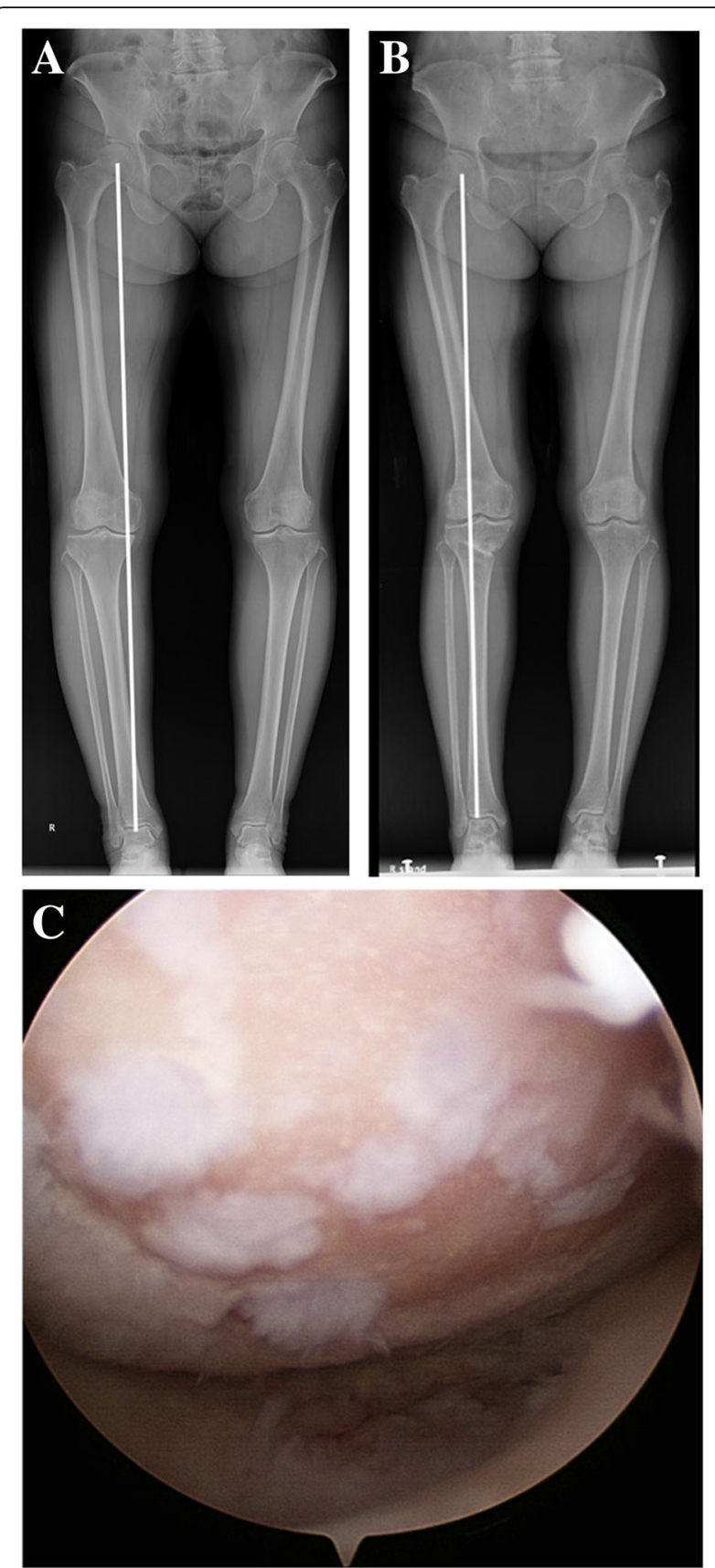

Fig. 2 Images from a 59-year-old woman show the following: (a) Ahlbäck grade I osteoarthritis with varus alignment of the lower limb before open wedge high tibial osteotomy with microfracture of the medial femoral condyle and medial tibial plateau; (b)

Postoperative alignment of the lower limb obtained a satisfactory WBL ratio (64.5\%). However, there was no recovery of the joint space narrowing; and (c) second-look arthroscopy shows insufficient coverage of the articular cartilage at postoperative 25 months. However, the Knee Society knee score significantly improved from 55 points preoperatively to 95 points, and the Western Ontario and McMaster Universities pain score significantly improved from 13 points preoperatively to 1 point at the time of second-look arthroscopy 

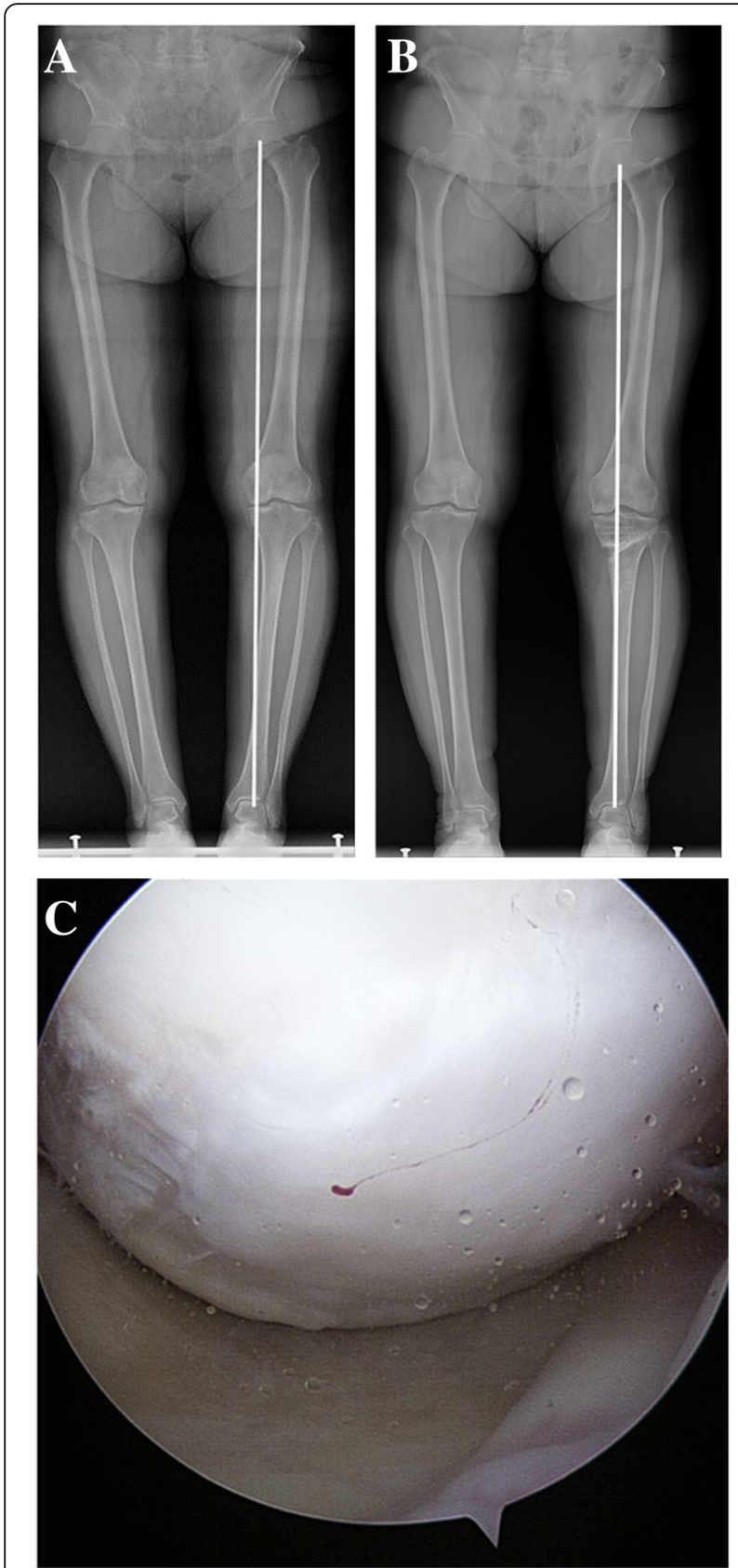

Fig. 3 Images from a 55-year-old woman show the following: (a) Ahlbäck grade I osteoarthritis with varus alignment of the lower limb before open wedge high tibial osteotomy with microfracture of the medial femoral condyle and medial tibial plateau; (b) Postoperative alignment of the lower limb was unsatisfactory (WBL ratio, 47.1\%); (c) Second-look arthroscopy shows sufficient restoration of the articular cartilage at postoperative 23 months. The postoperative Knee Society knee and function scores were 55 and 60 points, respectively, and the postoperative Western Ontario and McMaster Universities total score was 29 points, all of which were unsatisfactory regeneration did not agree with the improvement in clinical outcome after OW HTO with microfrature, have been identified (Figs. 2 and 3).

In terms of the MOCART score after HTO, Verdonk et al. [32] reported that complete integration to the border zone was found in $25 \%$ of patients at postoperative 2 years. However, Kim et al. [17] reported complete integration in 1 patient out of 14 patients with microfracture alone at 1-year follow-up. Our study also showed very low incidence of integration, which was consistent with the findings of Kim et al. (Table 3). Kim et al. [17] reported no hypertrophy of the cartilage, and subchondral bone marrow edema was found in $78.6 \%$ of patients with OW HTO plus microfracture. Conversely, in our study, hypertrophy of the cartilage was observed in $28.1 \%$ of patients, whereas subchondral bone marrow edema was found in only $29.8 \%$ of patients with OW HTO plus microfracture. These results could be attributed to the fact that the follow-up period of our study was 2 years, which was markedly longer than the 1-year follow-up of the study by Kim et al.

There are several limitations in this study. First, the follow-up period was only 2 years after surgery. A long-term investigation is necessary to determine whether the changes in articular cartilage affect survival after OW HTO. Second, a comparison has not been performed between the under-correction and over-correction groups. Degree of the correction could affect the biological regeneration of the articular cartilage after OW HTO due to the different loading on the medial compartment. However, there were few outliers under $55 \%$ or over $70 \%$ of the WBL ratio, so we assumed that degree of the correction would have little effect on the results. Third, the postoperative rehabilitation protocol differed between the patients with and without a microfracture. The differences in the timing of postoperative weight-bearing could affect the regeneration of cartilage.

\section{Conclusion}

Microfracture of the MFC during OWHTO only helped the filling of the degenerative cartilage defect and the integration of the cartilage with adjacent cartilage. However, the clinical and radiologic outcome could not be improved by mircrofracture in the OWHTO.

\footnotetext{
Abbreviations

HKA: hip-knee-ankle; HTO: high tibial osteotomy; ICRS: International Cartilage Repair Society; JLCA: joint line convergence angle; KS: Knee Society; LFC: lateral femoral condyle; LTP: lateral tibial plateau; MFC: medial femoral condyle; MOCART: magnetic resonance observation of cartilage repair tissue; MRI: magnetic resonance imaging; MTP: medial tibial plateau; OW: open wedge; SD: standard deviation; WBL: weight-bearing line; WOMAC: Western Ontario and McMaster University
} 


\section{Funding}

This work was supported by Basic Science Research Program through the Ministry of Education of the Republic of Korea and National Research Foundation of Korea (NRF) (NRF-2017R1D1A1A09000509).

\section{Availability of data and materials}

All data generated or analysed during this study are included in this published article.

\section{Authors' contributions}

YSL \& OSL designed this paper and performed critical revision of the manuscript. YSL coordinated the clinical study and OSL initiated and performed the study, analyzed the data, and wrote the manuscript. SJM \& $\mathrm{SHL}$ helped to draft the manuscript and all authors read and approved the final manuscript.

\section{Ethics approval and consent to participate}

This article was performed under the IRB permission (Seoul National University Bundang Hospital Institutional Review Board No: B-1611/370-303) and informed consent was waived due to the retrospective nature of this study.

\section{Consent for publication}

All presented cases in this report had written consents for publication.

\section{Competing interests}

The authors declare that they have no competing interests.

\section{Publisher's Note}

Springer Nature remains neutral with regard to jurisdictional claims in published maps and institutional affiliations.

\section{Author details}

${ }^{1}$ Department of Orthopaedic Surgery, Mediplex Sejong Hospital, Incheon, South Korea. ${ }^{2}$ Department of Orthopaedic Surgery, Incheon Metropolitan City Medical Center, Incheon, South Korea. ${ }^{3}$ Department of Orthopaedic Surgery, Seoul National University College of Medicine, Bundang Hospital, Seoul, South Korea.

Received: 7 October 2018 Accepted: 1 May 2019

Published online: 01 June 2019

\section{References}

1. Gaasbeek RD, Nicolaas L, Rijnberg WJ, van Loon Cl, van Kampen A. Correction accuracy and collateral laxity in open versus closed wedge high tibial osteotomy. A one-year randomised controlled study. Int Orthop. 2010;34:201-7.

2. Hernigou P, Medevielle D, Debeyre J, Goutallier D. Proximal tibial osteotomy for osteoarthritis with varus deformity. A ten to thirteen-year follow-up study. J Bone Joint Surg Am. 1987;69:332-54.

3. Koshino T, Murase T, Saito T: Medial opening-wedge high tibial osteotomy with use of porous hydroxyapatite to treat medial compartment osteoarthritis of the knee. J Bone Joint Surg Am 2003; 85-A:78-85.

4. Maurer F, Wassmer G. High tibial osteotomy: does navigation improve results? Orthopedics. 2006;29:S130-2.

5. Briem K, Ramsey DK, Newcomb W, Rudolph KS, Snyder-Mackler L. Effects of the amount of valgus correction for medial compartment knee osteoarthritis on clinical outcome, knee kinetics and muscle co-contraction after opening wedge high tibial osteotomy. Journal of orthopaedic research: official publication of the Orthopaedic Research Society. 2007;25:311-8

6. Prodromos CC, Andriacchi TP, Galante JO. A relationship between gait and clinical changes following high tibial osteotomy. J Bone Joint Surg Am. 1985;67:1188-94

7. Akizuki S, Yasukawa Y, Takizawa T. Does arthroscopic abrasion arthroplasty promote cartilage regeneration in osteoarthritic knees with eburnation? A prospective study of high tibial osteotomy with abrasion arthroplasty versus high tibial osteotomy alone. Arthroscopy: the journal of arthroscopic \& related surgery : official publication of the Arthroscopy Association of North America and the international arthroscopy association. 1997;13:9-17.

8. Jung WH, Takeuchi R, Chun CW, Lee JS, Ha JH, Kim JH, Jeong JH. Second-look arthroscopic assessment of cartilage regeneration after medial opening-wedge high tibial osteotomy. Arthroscopy: the journal of arthroscopic \& related surgery : official publication of the Arthroscopy Association of North America and the international arthroscopy association. 2014;30:72-9.

9. Koshino T, Wada S, Ara Y, Saito T. Regeneration of degenerated articular cartilage after high tibial valgus osteotomy for medial compartmental osteoarthritis of the knee. Knee. 2003;10:229-36.

10. Tang WC, Henderson IJP. High tibial osteotomy: long term survival analysis and patients' perspective. Knee. 2005;12:410-3.

11. McClelland D, Barlow D, Moores TS, Wynn-Jones C, Griffiths D, Ogrodnik PJ, Thomas PB. Medium- and long-term results of high tibial osteotomy using Garches external fixator and gait analysis for dynamic correction in varus osteoarthritis of the knee. The bone \& joint journal. 2016;98-b:601-7.

12. Koh YG, Kwon OR, Kim YS, Choi YJ. Comparative outcomes of open-wedge high tibial osteotomy with platelet-rich plasma alone or in combination with mesenchymal stem cell treatment: a prospective study. Arthroscopy: the journal of arthroscopic \& related surgery: official publication of the Arthroscopy Association of North America and the international arthroscopy association. 2014;30:1453-60

13. Wong KL, Lee KB, Tai BC, Law P, Lee EH, Hui JH. Injectable cultured bone marrow-derived mesenchymal stem cells in varus knees with cartilage defects undergoing high tibial osteotomy: a prospective, randomized controlled clinical trial with 2 years' follow-up. Arthroscopy: the journal of arthroscopic \& related surgery : official publication of the Arthroscopy Association of North America and the international arthroscopy association. 2013:29:2020-8

14. Matsunaga D, Akizuki S, Takizawa T, Yamazaki I, Kuraishi J. Repair of articular cartilage and clinical outcome after osteotomy with microfracture or abrasion arthroplasty for medial gonarthrosis. Knee. 2007;14:465-71.

15. Franceschi F, Longo UG, Ruzzini L, Marinozzi A, Maffulli N, Denaro V. Simultaneous arthroscopic implantation of autologous chondrocytes and high tibial osteotomy for tibial chondral defects in the varus knee. Knee. 2008;15:309-13.

16. Ferruzzi A, Buda R, Cavallo M, Timoncini A, Natali S, Giannini S. Cartilage repair procedures associated with high tibial osteotomy in varus knees: clinical results at 11 years' follow-up. Knee. 2014;21:445-50.

17. Kim MS, Koh IJ, Choi YJ, Pak KH, In Y. Collagen augmentation improves the quality of cartilage repair after microfracture in patients undergoing high Tibial osteotomy: a randomized controlled trial. Am J Sports Med. 2017:45:1845-55.

18. Pascale W, Luraghi S, Perico L, Pascale V. Do microfractures improve high tibial osteotomy outcome? Orthopedics. 2011;34:e251-5.

19. Lee YS, Lee OS, Lee SH: Effect of the microfracture on the regeneration of the cartilage and clinical outcome in the open wedge high tibial osteotomy. Knee Surg Sports Traumatol Arthrosc 2018; suppl 26:S5-S126.

20. Petersson IF, Boegård T, Saxne T, Silman AJ, Svensson B. Radiographic osteoarthritis of the knee classified by the Ahlbäck and Kellgren \& Lawrencesystems for the tibiofemoral joint in people aged 35-54 years with chronic knee pain. Ann Rheum Dis. 1997;56:493-6.

21. Lee YS, Kang JY, Lee MC, Oh WS, Elazab A, Song MK. Effect of the osteotomy length on the change of the posterior Tibial slope with a simple distraction of the posterior gap in the Uni- and Biplanar open-wedge high Tibial osteotomy. Arthroscopy: the journal of arthroscopic \& related surgery official publication of the Arthroscopy Association of North America and the international arthroscopy association. 2016;32:263-71.

22. Brittberg M, Winalski CS. Evaluation of cartilage injuries and repair. J Bone Joint Surg Am. 2003:85:58-69.

23. Marlovits S, Singer P, Zeller P, Mandl I, Haller J, Trattnig S. Magnetic resonance observation of cartilage repair tissue (MOCART) for the evaluation of autologous chondrocyte transplantation: determination of interobserver variability and correlation to clinical outcome after 2 years. Eur J Radiol. 2006:57:16-23.

24. Sanghvi D, Munshi M, Pardiwala D. Imaging of cartilage repair procedures. The Indian journal of radiology \& imaging. 2014;24:249-53.

25. Jung WH, Takeuchi R, Chun CW, Lee JS, Jeong JH. Comparison of results of medial opening-wedge high tibial osteotomy with and without subchondral drilling. Arthroscopy: the journal of arthroscopic \& related surgery : official publication of the Arthroscopy Association of North America and the international arthroscopy association. 2015;31:673-9.

26. Fujisawa $Y$, Masuhara K, Shiomi S. The effect of high tibial osteotomy on osteoarthritis of the knee. An arthroscopic study of 54 knee joints. Orthop Clin North Am. 1979;10:585-608.

27. Wakabayashi S, Akizuki S, Takizawa T, Yasukawa Y. A comparison of the healing potential of fibrillated cartilage versus eburnated bone in osteoarthritic knees after high tibial osteotomy: an arthroscopic study with 
1-year follow-up. Arthroscopy: the journal of arthroscopic \& related surgery : official publication of the Arthroscopy Association of North America and the international arthroscopy association. 2002;18:272-8.

28. Sterett WI, Steadman JR, Huang MJ, Matheny LM, Briggs KK. Chondra resurfacing and high tibial osteotomy in the varus knee: survivorship analysis. Am J Sports Med. 2010;38:1420-4.

29. Kim KI, Seo MC, Song SJ, Bae DK, Kim DH, Lee SH. Change of chondral lesions and predictive factors after medial open-wedge high Tibial osteotomy with a locked plate system. Am J Sports Med. 2017;45:1615-21.

30. Spahn G, Kirschbaum S, Kahl E. Factors that influence high tibial osteotomy results in patients with medial gonarthritis: a score to predict the results. Osteoarthritis and cartilage / OARS, Osteoarthritis Research Society. 2006;14:190-5.

31. Bonasia DE, Dettoni F, Sito G, Blonna D, Marmotti A, Bruzzone M, Castoldi F, Rossi R. Medial opening wedge high tibial osteotomy for medial compartment overload/arthritis in the varus knee: prognostic factors. Am J Sports Med. 2014;42:690-8.

32. Verdonk P, Dhollander A, Almqvist KF, Verdonk R, Victor J. Treatment of osteochondral lesions in the knee using a cell-free scaffold. The bone \& joint journal. 2015;97:318-23.

Ready to submit your research? Choose BMC and benefit from:

- fast, convenient online submission

- thorough peer review by experienced researchers in your field

- rapid publication on acceptance

- support for research data, including large and complex data types

- gold Open Access which fosters wider collaboration and increased citations

- maximum visibility for your research: over $100 \mathrm{M}$ website views per year

At $\mathrm{BMC}$, research is always in progress.

Learn more biomedcentral.com/submissions 